\title{
LA MEDICIÓN DEL DESARROLLO SOCIAL
}

\author{
Antonio Alaminos y Begoña López \\ Instituto Universitario de Desarrollo Social y Paz
}

\begin{abstract}
Resumen
Los índices de desarrollo han sido durante mucho tiempo objeto de debate, tanto académico como político o social. Es abundante la bibliografía que se ocupa de la noción de desarrollo social, tanto de su significado como de su dependencia histórica y cultural. Presentamos una descripción sintética de los índices más significativos, destacando las dimensiones teóricas así como sus especificidades metodológicas.
\end{abstract}

Palabras clave: desarrollo humano, índices, medio ambiente, calidad de vida.

\begin{abstract}
The indexes of development have been matter of political, academic and social discusión. There is abundant literature on the concept of social development, its meaning and its historical and cultural dependence. We present a summary description of the most significant indexes, highlighting the theoretical dimensions and their specific methodologies.
\end{abstract}

Keywords: human development, indexes, environment, quality of life.

\section{INTRODUCCIÓN}

La medición del desarrollo social plantea cuestiones centrales en tres ámbitos diferenciados. Por una parte existe un debate de carácter teórico, otro de naturaleza metodológica y finalmente, un conflicto ético.

Desde el punto de vista de la teoría, destacan dos estrategias complementarias. Por un lado, la ampliación del foco de interés, que se refleja tanto en la incorporación de nuevas dimensiones a la noción de desarrollo, como en el reconocimiento de nuevos sujetos sociales. En la línea de incrementar las dimensiones definitorias, aparecen propuestas de introducir aspectos relacionados con el consumo, el medio ambiente, la huella ecológica, etc., como 
parte de la idea de desarrollo. Los nuevos sujetos reflejan la evidente desigualdad y desequilibrio existente dentro de las sociedades. En ese sentido, la noción de desarrollo es una idea sesgada si exclusivamente se aplica a una unidad agregada (sea Estado, sociedad, etc.). Surge entonces la propuesta de desarrollo como convergencia futura; un punto de encuentro del desarrollo local de los diferentes sujetos que experimentan la desigualdad. Este desarrollo asimétrico obliga a reconocer sujetos y desigualdades como son género, etnia, edad, etc. En definitiva, el desarrollo debe partir como primera referencia empírica de los desequilibrios internos de toda sociedad.

Las dos líneas de acción mencionadas actúan en el sentido de incrementar la visibilidad de los sujetos del desarrollo social y de los aspectos que puedan formar parte de la definición. En ese sentido, incorporando el reconocimiento de los desequilibrios y la desigualdad mejoran sensiblemente la noción de desarrollo social.

La segunda estrategia estudia directamente la noción de desarrollo. La concepción de fondo presupone que el desarrollo es un objetivo teóricamente abierto pero empíricamente cerrado. En definitiva, los límites empíricos siempre han estado presentes tras un desarrollo teóricamente abierto. Posiblemente el concepto de desarrollo es el que más debate produce en el contexto de elaborar un indicador. Entre las respuestas destacan las propuestas que hablan de equilibrio. Una vez que la naturaleza y su deterioro (contaminación, hambrunas, sequías, enfermedades por exposiciones a productos tóxicos, etc.) han evidenciado con claridad los límites empíricos. Más evidentemente que nunca, se impone la percepción de que desarrollo no es necesariamente un sinónimo de crecimiento. Las propuestas alternativas proponen como sinónimo de desarrollo la búsqueda de equilibrio. Considerada con detenimiento, la idea de desarrollo sostenible es en gran parte un eufemismo para hablar de equilibrio. Un eufemismo que no pierde los viejos hábitos pero que ya habla de una realidad diferente. Equilibrio con el medio ambiente y equilibrio social interno (atenuación de las desigualdades). Esto se evidencia en las propuestas que plantean incluso la necesidad de decrecimiento, precisamente para recuperar la posibilidad de equilibrio ecológico. Asistimos a una nueva reinterpretación donde la noción de desarrollo se desvincula de la idea de crecimiento, proponiendo contenidos nuevos.

Desde el punto de vista metodológico, uno de los aspectos más interesantes es la necesidad de "cualificar lo cuantitativo y cuantitivizar lo cualitativo". En definitiva conocer los estados internos de los individuos en relación a la calidad de la vida que viven. Son las propuestas que hacen énfasis en los aspectos psicológicos, como son por ejemplo la felicidad. En ese sentido, el desa- 
rrollo social debería contener la percepción que los individuos tienen de su propia vida. Parece razonable que el desarrollo social incorpore, además de indicadores relativamente objetivos o externos, indicadores subjetivos.

Existen, sin embargo, varios problemas asociados a dicho enfoque metodológico. Por un lado, la reducción drástica a las posibilidades de comparación entre culturas y definiciones de la felicidad diferentes. La idea de buen vivir (desprendida de sus raíces como referencia a una ética extensa) incorpora elementos subjetivos (de los que solamente puede informar el individuo) de primer nivel. Esta noción, positiva en si misma, exige de matizaciones muy importantes. En primer lugar, el acceso del sujeto (sociedad) a la interpretación del concepto nos obliga a emplear la distinción entre la perspectiva Etic y la perspectiva Emic. La perspectiva Emic nos remite a la visión que los individuos tienen de su propia vida. Las motivaciones y las razones que emplean para explicarse a si mismos y a los demás. En definitiva, una historia narrada en primera persona. Un narrador intradiegético que cuenta y protagoniza su propia historia vital. Un enfoque diferente consiste en la perspectiva Etic. En esta interpretación, el observador permanece fuera de la historia. Su opinión aparece ampliando, corrigiendo e interpretando la vida que viven los demás. En este enfoque, el investigador adopta idealmente la posición de narrador extradiegético. No participa ni se identifica con los hechos (sean comportamientos, valores u opiniones, por ejemplo) que estudia. Gran parte de la historia del desarrollo social es una narración Etic, donde la medición se efectuaba de modo externalizado a la sociedad que estudia. El PIB como indicador de desarrollo es un buen ejemplo de narración extradiegética. La incorporación de dimensiones subjetivas obliga a una integración "controlada" de las percepciones Emic.

Entre las razones, la posibilidad de una sociedad que desde la perspectiva Etic se encuentre semiesclavizada. Tanto globalmente, como para diferentes sujetos sociales; por ejemplo la posición social subordinada de la mujer en muchas culturas. Un autodiagnóstico Emic de felicidad puede ser incompatible con la idea Etic de desarrollo social. Hemos dado con uno de los conceptos clave de la sociología; la alienación. En su definición más simple, la percepción alterada de la realidad. En los términos aquí empleados, la critica Etic a las posiciones Emic se basaría en una incompatibilidad entre la felicidad y las condiciones objetivas en las que se ejerce.

Los conceptos Etic y Emic fueron propuestos por el lingüista Pike y tomados en sus propuestas metodológicas por M. Harris. La diferencia fundamental entre ambos investigadores es la posible jerarquización entre enfoques. Para Pike, tanto los enfoques Etic como Emic son correctos y no existe más verdad 
en uno que en el otro. En definitiva el concepto de alienación pierde su carácter de puente conector entre la realidad Etic y la interpretación intradiegética de los sujetos. Para M. Harris, el enfoque Etic es superior a la interpretación Emic. Es decir, existe un ámbito con un menor grado de subjetividad desde el que se puede valorar el grado de realidad (consistencia entre lo que se hace, la razón por lo que se hace y lo que realmente se logra) de las acciones sociales. En esta percepción ordinal de dos enfoques, el concepto de alienación adquiere un significado pleno.

Nos encontramos pues ante dos posiciones metodológicas diferentes. En la primera (Pike), una vez introducidos elementos subjetivos en el índice de desarrollo, llegamos a un pluralismo incomparable. Los desarrollos son autóctonos y autorreferenciados. La segunda posición metodológica (Harris) nos conduce a un concepto de desarrollo social dentro de los parámetros científicos conocidos. Es decir, un índice normalizado y que permite la comparación dentro y entre sociedades. Entre otros, acorde con los criterios de precisión, disponibilidad, simplicidad, fiabilidad, validez, especificidad, confiabilidad, sensibilidad y alcance. Hay, sin embargo, un pago que hacer por tal decisión de normalización. Y no es un pago menor.

La superación de las diferentes raíces culturales de la felicidad exige de un marco de referencia. Podría ser cualquiera, incluyendo además de culturas, éticas religiosas o civiles. ¿Cual puede ser el pivote de referencia de un desarrollo/equilibrio social? La respuesta esta incluida en la misma pregunta. Si el "desarrollo social" lo hemos definimos como equilibrio económico, social y ecológico (entendiendo las desigualdades como desequilibrios) la respuesta son los derechos humanos ${ }^{1}$. Herencia de la ilustración europea, de la revolución francesa y producto histórico, ciertamente etnocéntricos (al igual que lo es la Ley del Talión, por ejemplo), pero enfocados en lo humano. En el hombre. En ese sentido, la carta de derechos humanos debería ser el fundamento, el grado cero de cualquier índice que quiera hablar de "desarrollo social".

Como hemos visto, la definición misma de desarrollo como equilibrio nos conduce a un enfoque particular. Sin embargo, es inevitable la aparición de elementos axiológicos culturales en cualquier índice de desarrollo que incorpore elementos subjetivos. El etnocentrismo es un requisito y una consecuencia del enfoque teórico (introducción de los subjetivo) y metodológico (comparabilidad y generalización). Además, es una opción axiológica.

No obstante, el hecho de ser etnocéntrico en lo que se refiere a los derechos humanos, no es un vicio de origen en cualquier indicador. A pesar de que ya no pueda hablarse de un índice desvinculado de proyectos de legiti-

1. Declaración Universal de los Derechos Humanos. http://www.un.org/es/documents/udhr/ 
mación del poder. Sin embargo, si excluye la legitimación de cualquier proyecto de poder local. Especialmente los que abandonan el principio "societario" por el "comunitario". Esta es una de las dimensiones más sensibles en las diferentes propuestas y modificaciones.

Vamos a presentar someramente algunas de las propuestas existentes en la medición del desarrollo social (en sus múltiples sinónimos). En concreto.

- Índice de Desarrollo Humano

- Índice de Desarrollo Humano relativo al género.

- Índice de Pobreza Humana

- Índice Genuino del Progreso (similar al Índice de Bienestar Económico Sostenible)

- Índice del Planeta Feliz

- Felicidad Interna Bruta

\section{1. ÍNDICE DE DESARROLLO HUMANO}

El IDH nace en 1990 en un informe presentando en el PNUD (Programa de las Naciones Unidas para el desarrollo) el Human Development Report (Informe sobre Desarrollo Humano) que se ha ido elaborando anualmente hasta la actualidad. Marca un punto y a parte en la visión del desarrollo, al aplicar una nueva concepción del mismo, considerando su estudio como algo mas allá de las fronteras económicas. Se desbanca el PIB como instrumento único de medición del bienestar. Se trata de un índice que identifica la situación de un país en cuanto al desarrollo humano básico de su población mediante un índice compuesto.

Entre sus precursores podemos encontrar a Amartya Sen con su aportación sobre la teoría de las capacidades, que ya en la década de los ochenta incidió en la necesidad de enfocar los estudios de bienestar en las capacidades y habilidades personales de la gente ${ }^{2}$ o Rawls y la "Teoría de la Justicia", en la que la privación se define en términos de disponibilidad de bienes primarios, unos se refieren a bienes materiales, otros a libertades básicas.

Otras referencias podemos encontrarlas en la década de los 70 , en la propuesta de la OIT (Organización Internacional del Trabajo) a través del "Enfoque de las necesidades básicas", o de Max Neef y su teoría del "Desarrollo a escala humana", en la que define una matriz que abarca 9 necesidades humanas básicas: subsistencia, protección, afecto, comprensión, participación, creación, recreo, identidad y libertad.

2. Construcción y análisis del índice de calidad de vida en Guatemala. Berumen, Sergio. Estudios Económicos de Desarrollo Internacional. Vol. 4-2 (2004) 


\section{Composición:}

El IDH Consta de tres elementos fundamentales que determinan el desarrollo de una sociedad. Los tres tienen el mismo peso sobre el índice, de esta manera se evita la infravaloración de cualquiera de los elementos. Cada uno tiene distintos valores máximos y mínimos en función de las características.

- Salud

- Educación

- Renta.

Los valores máximos y mínimos para cada uno son los siguientes: esperanza de vida al nacer con un valor máximo de 85 años y mínimo de 25 , el rango de la educación oscila entre 0 y 100\% y, por último, el PIB per cápita que oscila entre los 40.000 y 100 dólares. En el siguiente cuadro se muestran los distintos componentes del Índice de Desarrollo Humano junto a los indicadores utilizados para cada uno de ellos y los índices resultantes:

Tabla 1: Componentes, indicadores e índices del IDH

\begin{tabular}{l|l|l}
\hline \multicolumn{1}{c|}{ Componente } & \multicolumn{1}{c}{ Indicador } & \multicolumn{1}{c}{ Indice } \\
\hline Vida larga y saludable & $\begin{array}{l}\text { Esperanza de vida } \\
\text { al nacer }\end{array}$ & $\begin{array}{l}\text { Indice de Esperanza } \\
\text { de vida }\end{array}$ \\
\hline Educación & $\begin{array}{l}\text { 1. Tasa de alfabetización } \\
\text { de adultos }\end{array}$ & $\begin{array}{l}\text { Índice de Alfabetización } \\
\text { de adultos }\end{array}$ \\
\hline Nivel de vida digno & $\begin{array}{l}\text { 2. Tasa Bruta de } \\
\text { matriculación }\end{array}$ & $\begin{array}{l}\text { Índice de la Tasa Bruta } \\
\text { de matriculación }\end{array}$ \\
\hline
\end{tabular}

En la fórmula del IDH se contabiliza pues, la esperanza de vida, la educación -resultante de la combinatoria entre alfabetización y matriculación-, y el PIB per. cápita ${ }^{3}$.

- a través de una corrección del PIB que considera las diferencias de precios entre países reflejando de mejor modo el nivel de vida de las personas.

Siendo su fórmula la siguiente:

$$
\mathrm{IDH}=1 / 3(\mathrm{IEV})+1 / 3(\mathrm{IE})+1 / 3(\mathrm{IPBI}) \text {, donde: }
$$

3. Para más información sobre el Índice de Desarrollo Humano consultar en la Web del PNUD: http://hdr.undp.org/es/estadisticas/indices/idh/ 
IEV: Esperanza de vida al nacer

IE: Índice de Educación

IA: Índice de Alfabetización Adulta

IM: Índice Bruto de Matriculación

IPBI: Índice del Producto Interior Bruto

La valoración del resultado final de este indicador sigue una progresión de bajo/medio/alto, tomando como referencia los siguientes valores:

IDH alto: mayor o igual que 0.8

IDH medio: entre 0.5 y 0.8

IDH bajo: mejor que 0.5

Una de las limitaciones que se le atribuye a este índice es que de año en año se cambian los criterios metodológicos utilizados para definir las variables básicas a partir de las que se elaboran los indicadores. Este cambio dificulta las comparaciones intertemporales, así como obtener unos resultados que se aproximen con mayor exactitud sobre el avance o el retroceso en términos de desarrollo humano.

\section{2. ÍNDICE DE DESARROLLO HUMANO RELATIVO AL GÉNERO}

Esta medida reduce el Índice de Desarrollo Humano por inequidades de género en las tres dimensiones cubiertas por el IDH, considerando y cuantificando las desigualdades sociales y económicas entre hombres y mujeres. Fue introducido en el Informe sobre Desarrollo Humano de 1995 junto al índice de potenciación de género (IPG).

\section{Composición:}

El IDHG mide los logros en las mismas dimensiones que el IDH pero teniendo en cuenta la dimensión de género y de esa manera es sensible a las desigualdades de género. Así, en este índice se considera la dimensión de vida larga y saludable -esperanza de vida al nacer de cada sexo-, educación -combinatoria de la tasa de alfabetización de adultos y la tasa bruta combinada de matriculación en educación primaria, secundaria y terciaria por sexo y, por último nivel de vida digno- estimado a partir de los ingresos por sexo. Al igual que con el IDH, a continuación se detallan los distintos componentes, indicadores e índices para cada uno de ellos. 
Tabla 2: Componentes, indicadores e índices del IDHG

\begin{tabular}{l|l|l}
\hline \multicolumn{1}{c|}{ Componente } & \multicolumn{1}{c}{ Indicador } & \multicolumn{1}{c}{ Indice } \\
\hline Esperanza de vida & $\begin{array}{l}\text { 1. Esperanza de vida de las } \\
\text { mujeres } \\
\text { 2. Esperanza de vida de los } \\
\text { de vida de hombres }\end{array}$ & $\begin{array}{l}\text { 1. Índice de esperanza de } \\
\text { vida de mujeres } \\
\text { 2. Índice de esperanza } \\
\text { de vida de hombres } \\
\text { Índice esperanza de vida } \\
\text { igualmente }\end{array}$ \\
\hline Educación & $\begin{array}{l}\text { 1. Tasa de alfabetización de } \\
\text { mujeres } \\
\text { 2. Tasa de alfabetización de } \\
\text { hombres }\end{array}$ & $\begin{array}{l}\text { 1. Índice de educación } \\
\text { de mujeres } \\
\text { 2. Índice de educación } \\
\text { de hombres } \\
\text { Índice de Educación } \\
\text { igualmente distribuido }\end{array}$ \\
\hline Nivel de vida digno & $\begin{array}{l}\text { 1. Cálculo de ingresos } \\
\text { percibidos por mujeres } \\
\text { 2. Cálculo de ingresos } \\
\text { percibidos por hombres }\end{array}$ & $\begin{array}{l}\text { 1. Índice de ingresos } \\
\text { de mujeres } \\
\text { 2. Índice de ingresos } \\
\text { de hombres } \\
\text { Índice de Educación } \\
\text { igualmente distribuido }\end{array}$ \\
\hline
\end{tabular}

\section{3. ÍNDICE DE POBREZA HUMANA}

En 1996 tiene lugar en el PNUD el primer intento de medir las situaciones de pobreza con el fin de introducir una nueva perspectiva cuantitativa en los estudios de desarrollo. Es así como surge en un primer momento el Índice de Pobreza de Capacidad ${ }^{4}$ (IPC), un índice compuesto que medía a través de tres dimensiones básicas el desarrollo humano.

De esta manera, estar alfabetizado y tener acceso al conocimiento, gozar de una alimentación adecuada y poder dar a luz sin riesgo y en buenas condiciones será tenido en cuenta para la contabilidad de la pobreza, obteniendo como resultado la siguiente ecuación:

Cuadro 1: Cálculo de Índice de Pobreza de Capacidades

IPC $=(\%$ mujeres analfabetas $+\%$ niños menores de 5 años con peso insuficiente $+\%$ de nacimientos no atendidos con personal de salud no experimentado) / 3

4. Desarrollo Humano: Origen., Evolución e Impacto. Keith Griffin. 
Al año siguiente, 1997, fue cuando el PNUD plantea por primera vez el Índice de Pobreza Humana (IPH) en un intento de mejorar el anterior. El número de variables consideradas se amplió a 5 y se produjo una selección de indicadores relativos a la privación que se asemejaran, en la medida de lo posible, a los planteados en el IDH.

Si bien el IDH se centra en el progreso de una comunidad en su conjunto, el fenómeno de la pobreza se concentra en la situación y el progreso del conjunto de habitantes de una dentro de una comunidad, aquellos que sufren mayores privaciones que el resto. El Índice de Desarrollo Humano no estudia de manera directa las situaciones de pobreza, por lo que resulta más enriquecedora la combinación de este con el IPH, una medición multidimensional de la pobreza.

La pobreza puede analizarse desde tres perspectivas ${ }^{5}$ :

Perspectiva del ingreso: Un persona se considera pobre cuando su nivel de ingreso es inferior a la línea de pobreza que se ha definido.

Perspectiva de las necesidades básicas: la pobreza es entendida como la privación de los medios materiales para satisfacer en una medida minimamente aceptable las necesidades humanas (perspectiva que va más allá de la falta de ingreso)

Despectiva de capacidad: Ausencia de ciertas capacidades básicas necesarias para que una persona pueda funcionar. Se considera pobre una persona que carece de oportunidades para alcanzar unos límites minimamente aceptables.

El Índice de Pobreza Humana mantiene una relación inversa al Índice de Desarrollo Humano, al centrarse principalmente en las privaciones: supervivencia -vulnerabilidad a la muerte en una edad relativamente temprana-; conocimiento -quedar excluido de la lectura y las comunicaciones, medida a través del porcentaje de adultos- $\mathrm{y}$ un nivel de vida decente -aprovisionamiento económico general-. Mientras que una puntuación alta del IDH implica un elevado desarrollo, en el caso del IPH, los valores altos implican una situación de pobreza mayor.

Existen dos tipos de índices en función del país cuyo nivel de pobreza se quiera estudiar. El Índice de Pobreza Humana 1 se aplica para los países en vías de desarrollo, mientras que el Índice de Pobreza Humana 2 está destinado al estudio de situaciones de pobreza en países desarrollados. (Selección de

5. Informe sobre Desarrollo Humano 1997. Capítulo 1. La pobreza en la perspectiva del desarrollo humano: Concepto y medición. 
países de la OCDE). La diferencia entre ambos radica principalmente en que el segundo introduce la variable exclusión social a través de la tasa de desempleo a largo plazo. Por otro lado, en la probabilidad de morir a una edad relativamente temprana, se pasa de 40 a 60 años $\mathrm{Y}$ en el de vida digna se introduce el porcentaje de personas que viven por debajo del umbral de la pobreza. De este modo se introduce la perspectiva de la pobreza relativa, que implica la definición de una situación de pobreza, no puede ser la misma en un país en vías de desarrollo que en uno desarrollado.

A continuación aparecen los componentes, indicadores e índices para los dos.

Tabla 3 Componentes, indicadores e índices del IPHI

\begin{tabular}{l|l}
\hline \multicolumn{1}{c|}{ Componente } & \multicolumn{1}{c}{ Indicador } \\
\hline Vida larga y saludable & 1. Probabilidad al nacer de no vivir hasta los 40 años \\
\hline Educación & 1. Tasa de analfabetismo de adultos \\
\hline Nivel de vida digno & $\begin{array}{l}\text { 1. Porcentaje de la población sin acceso sostenible a } \\
\text { fuente de agua } \\
\text { 2. Porcentaje de niños con peso insuficiente para su edad }\end{array}$ \\
\hline
\end{tabular}

Tabla 4: Componentes, indicadores e índices del IPH 2

\begin{tabular}{l|l}
\hline \multicolumn{1}{c|}{ Componente } & \multicolumn{1}{c}{ Indicador } \\
\hline Vida larga y saludable & 1. Probabilidad al nacer de no vivir hasta los 60 años \\
\hline Educación & $\begin{array}{l}\text { 1. Porcentaje de adultos que carecen de aptitudes de } \\
\text { alfabetización funcional }\end{array}$ \\
\hline Nivel de vida digno & $\begin{array}{l}\text { 1. Porcentaje personas que viven por debajo del } \\
\text { umbral de pobreza }\end{array}$ \\
\hline Exclusión social & 1. Tasa de desempleo a largo plazo \\
\hline
\end{tabular}

Como se comentó en el párrafo anterior, la tasa de empleo a largo plazo se introduce en la elaboración del Índice de Pobreza Humana para los países desarrollados, mientras que no se encuentra dentro del primero. Por último, resaltar que los valores del IPH, al igual que los del Índice de Desarrollo Humano, oscilan entre 0 y 100.

\section{4. ÍNDICE DE PROGRESO GENUINO (IPG)}

El Índice del progreso genuino surge como alternativa al PIB en calidad de indicador de bienestar social. Creado por un grupo de investigadores de California 
en la década de los 70, a diferencia del PIB, distingue entre las actividades económicas que producen beneficios y las que causan daño. Esto supone un cambio de perspectiva importante, no todas las actividades generar beneficio común.

En el cuadro que se presenta a continuación aparece el cálculo que se utiliza para la elaboración del Índice de Progreso Genuino. Para realizar el cálculo se toma como punto de partida los datos de consumo personal -ajustados en función de de la distribución de ingresos-, a estos se les añade los valores del tiempo de trabajo doméstico, y comunitario -cuidados familiares, voluntariado, y así hasta 26 variables más-. Por otra parte se descuentan los gastos defensivos -aquellos destinados a evitar o minimizar los efectos derivados de la inseguridad, los accidentes o la contaminación-, los "costes sociales" y la depreciación de los recursos naturales -valorados como reservas finitas de capital-. En total, el IPG, está compuesto por 26 variables de carácter social, económico y ambiental.

En el cuadro siguiente aparece representado de manera esquemática el cálculo para obtener el índice.

\section{Cuadro 2: Cálculo del Índice de Progreso Genuino (IPG)}

Consumo comercial doméstico ajustado en función de la desigualdad económica

Servicios de trabajo doméstico y comunitario

$+$

Gastos públicos

-

Gastos privados y públicos "defensivos"

Gastos de degradación del medio ambiente

Desvalorización del capital natural

Algunos de los componentes que analiza están relacionados con el trabajo -valor del trabajo voluntario y en el hogar-, el medio ambiente -valor del capital de los recursos naturales, costes de la contaminación ó destrucción del agotamiento de los mismos-, y variables relacionadas con la calidad de vida -valor de la salud de las personas, coste de la pérdida del tiempo libre, etc-. 
A continuación, y rompiendo la tendencia que se ha mantenido hasta el momento, se presentan dos índices que se basan principalmente en el análisis de variables, en su mayoría, subjetivas de interpretar, tales como la percepción de la satisfacción en la vida ó el nivel de felicidad de un país. Ambos llevan implícitos en si mismos una debilidad, la que conlleva el análisis y la posterior interpretación de variables subjetivas.

Tanto en el Índice del Planeta Feliz como en el de Felicidad Interna Bruta se considera la satisfacción en la vida, el bienestar individual y social, etc. Esto plantea un arma de doble filo, puesto que al mismo tiempo que supone un avance en el estudio del desarrollo humano, puede dar pie a la manipulación cuando los resultados obtenidos justifican o dificultan el cambio de situaciones en algunas sociedades que no presentan niveles de desarrollo satisfactorios. Como es el caso de Vanuatu, cuyos altos índices de pobreza se contraponen con su posición a la cabeza en la clasificación de países mas felices. A continuación se presentan las características principales de ambos índices.

\section{5. ÍNDICE DEL PLANETA FELIZ}

Creado por la Fundación Nueva Economía (NEF), surge de la necesidad de introducir los factores ambientales en el estudio del desarrollo de las sociedades y de las personas. Parte de la base de que sin un entorno sano no es posible un desarrollo completo. Se obtiene a través de una fórmula no matemática que considera tres dimensiones: Esperanza de vida, satisfacción en la vida -los dos primeros englobarían los "años felices de vida"- y la huella ecológica -relación entre calidad de vida y los recursos que se obtienen a través del planeta-. El cálculo para obtener los resultados seria el siguiente:

\section{Cuadro 3: Cálculo del Índice del Planeta Feliz.}

Esperanza de vida $x$ Satisfacción en la vida/ Huella ecológica

Se mide el número de planetas que serian necesarios como inversión ecológica. La media razonable que plantean los precursores del índice esta representado por un 8,0 para la satisfacción en la vida -en un intervalo en 0 y 10-, 87 años de esperanza de vida y una huella ecológica de 1,7. Al aplicar la fórmula propuesta, el Índice resultante sería de 89.

En julio de 2009 se elaboró el último informe sobre el Índice del Planeta Feliz ${ }^{6}$. En el, Vanuatu encabezaba la lista con un índice de 68,2, seguido de

6. Para mas información consultar la Web: the happy planet index 2.0 (happyplanetindex.org). 
Colombia $(67,2)$ y Costa Rica (66). En los últimos puestos se encuentran Zimbabwe $(16,6)$, Swazilandia $(18,4)$ y Burundi $(19)$. Países como Estados Unidos, Portugal ó Francia se sitúan en los puestos $150^{\circ}$; $136^{\circ}$ y $129^{\circ}$ respectivamente. España se encontraría en el puesto 76 , con índice final de 43,2 $-7,8$ en el índice relacionado con la satisfacción en la vida, 80,5 años de esperanza media y una huella ecológica de 5,7-

Cabe descatar resultados, a primera vista "curiosos", un segundo puesto ocupado por Bolivia -país que se situa en las primeras posiciones en la lista de países con desplazamientos forzados- y otros países como Portugal ó Francia por encima del puesto 100. Esto supone un cambio en la polaridad presente actualmente en el mundo en términos subjetivos de felicidad.

\section{FELICIDAD INTERNA BRUTA (FIB)}

El término fue propuesto por el Rey de Bután Jigme Singye Wangchuck en 1972, como respuesta a las críticas de la constante pobreza económica del país. El pasado mes de Noviembre se celebró el V Congreso Internacional sobre Felicidad Interna Bruta en Brasil, el Centro de Estudios Butaneses ${ }^{7}$ en el que se incidió en la importancia de establecer entre sus principales objetivos el de discutir diferentes aspectos relacionados con el desarrollo, estuvieron convocados lideres políticos, miembros de la sociedad civil, intelectuales y académicos.

El estudio se realiza a través de encuestas de percepción, construidas con una matriz de 150 indicadores en los que se integran las dimensiones anteriores. Establece nueve dimensiones para el estudio de las sociedades:

Bienestar psicológico: Evalúa el grado de satisfacción y optimismo en la vida individual. Los indicadores de análisis de la autoestima, sentido de competencia, el estrés, las actividades espirituales y la prevalencia de las emociones positivas y negativas.

Salud: Medidas de la eficacia de las políticas de salud, con criterios como la autovaloración, la discapacidad, las pautas de comportamiento de riesgo, ejercicio, sueño, nutrición, etc.

Uso del tiempo: Se evalúa a a través del estudio de la gestión del tiempo de los ciudadanos.

Vitalidad comunitaria: Analiza las relaciones e interacciones en las comunidades, a través del nivel de confianza, el sentido de pertenencia, la seguridad en el hogar y en la comunidad y la practica de voluntariado.

7. La información sobre el Congreso celebrado y otros aspectos relacionados con el Índice pueden consultarse en la Web del Centro de Estudios Butaneses: http://www.bhutanstudies.org.bt/main/gnhfive.php

8. Toda la información relacionada con la composición del Índice ha sido extraída de la página Web: www.felicidadeinternabruta.org.br 
Educación: Considera diversos factores como el nivel de participación en la educación, el desarrollo de habilidades y capacidades, la participación en la educación de los niños, la educación en valores, ambiental, etc.

Cultura: Evalúa las tradiciones locales, fiestas, valores y participación en eventos culturales. Así como las oportunidades de desarrollar habilidades artísticas y la discriminación en términos de religión o sexo.

Medio Ambiente: Mide la percepción de los ciudadanos sobre la calidad del agua, del aire, el suelo, la cubierta forestal, la biodiversidad, etc.

Gobierno: Evalúa la percepción por parte de la comunidad del gobierno, los medios de comunicación, el sistema judicial, electoral y policial. Y por último el nivel de vida, que evalúa de manera individual el ingreso familiar, las deudas contraídas, la seguridad en el empleo y la calidad de la vivienda.

Los defensores del Índice afirman que el FIB se basa en la premisa de que "El cálculo de la riqueza debe considerar otros aspectos además del desarrollo económico como la preservación del medio ambiente y la calidad de vida de la gente. El objetivo de una sociedad debe ser la integración del desarrollo material con el psicológico, cultural y los aspectos espirituales".

\section{BIBLIOGRAFÍA CONSULTADA:}

Castells, Manuel. Globalización, Identidad y Estado en América Latina. Temas de Desarrollo Humano Sustentable. PNUD Santiago. 1999.

Max -Neef, Manfred. Desarrollo a Escala Humana: Conceptos, Aplicaciones y Reflexiones Icara.1993.

Max - Neef, Manfred. Economía Descalca. Señales desde el Mundo Invisible 1993.

Rawls, Jhon. La Justicia como equidad: una reformulación. Paidós Ibérica, 2001.

Sen, Amartya. Desarrollo como libertad (Development as Freedom), Oxford, Oxford University Press, 1999.

Tortosa, José María. El juego global: maldesarrollo y pobreza en el capitalismo mundial. Icara. Barcelona. 2001.

Informe sobre desarrollo humano 2007-2008. Pág. 359-361.

Informe sobre Desarrollo Humano 1997. Capítulo 1. La pobreza en la perspectiva del desarrollo humano: Concepto y medición.

Schuschny, A. y Soto, H. Guía metodológica. Diseño de Indicadores compuestos de desarrollo sostenible. Comisión Económica para América Latina y el Caribe.

9. Extraido de la página Web: www.felicidadeinternabruta.org.br/en.html 